\title{
Restoration of Rostral Ventrolateral Medulla Cystathionine- $\gamma$ Lyase Activity Underlies Moxonidine-Evoked Neuroprotection and Sympathoinhibition in Diabetic Rats
}

\author{
Mohamed A. Fouda, Shaimaa S. El-Sayed, ${ }^{1}$ and Abdel A. Abdel-Rahman \\ Department of Pharmacology, Brody School of Medicine, East Carolina University, Greenville, North Carolina \\ Received July 13, 2017; accepted November 9, 2017
}

\begin{abstract}
We recently demonstrated a fundamental role for cystathionine- $\gamma$ lyase (CSE)-derived hydrogen sulfide $\left(\mathrm{H}_{2} \mathrm{~S}\right)$ in the cardioprotective effect of the centrally acting drug moxonidine in diabetic rats. Whether a downregulated CSE/ $\mathrm{H}_{2} \mathrm{~S}$ system in the rostral ventrolateral medulla (RVLM) underlies neuronal oxidative stress and sympathoexcitation in diabetes has not been investigated. Along with addressing this question, we tested the hypothesis that moxonidine prevents the diabetesevoked neurochemical effects by restoring $\mathrm{CSE} / \mathrm{H}_{2} \mathrm{~S}$ function within its major site of action, the RVLM. Ex vivo studies were performed on RVLM tissues of streptozotocin ( $55 \mathrm{mg} / \mathrm{kg}$, i.p.) diabetic rats treated daily for 3 weeks with moxonidine (2 or $6 \mathrm{mg} / \mathrm{kg}$; gavage), $\mathrm{H}_{2} \mathrm{~S}$ donor sodium hydrosulfide (NaHS) (3.4 $\mathrm{mg} / \mathrm{kg}$, i.p.), CSE inhibitor DL-propargylglycine (DLP) $(37.5 \mathrm{mg} / \mathrm{kg}$, i.p.), a combination of DLP with moxonidine, or
\end{abstract}

their vehicle. Moxonidine alleviated RVLM oxidative stress, neuronal injury, and increased tyrosine hydroxylase immunoreactivity (sympathoexcitation) by restoring CSE expression/ activity as well as heme oxygenase-1 (HO-1) expression. A pivotal role for $\mathrm{H}_{2} \mathrm{~S}$ in moxonidine-evoked neuroprotection is supported by the following: 1) NaHS replicated the moxonidineevoked neuroprotection, and the restoration of RVLM HO-1 expression in diabetic rats; and 2) DLP abolished moxonidineevoked neuroprotection in diabetic rats, and caused RVLM neurotoxicity, reminiscent of a diabetes-evoked neuronal phenotype, in healthy rats. These findings suggest a novel role for RVLM CSE/ $\mathrm{H}_{2} \mathrm{~S} / \mathrm{HO}-1$ in moxonidine-evoked neuroprotection and sympathoinhibition, and as a therapeutic target for developing new drugs for alleviating diabetes-evoked RVLM neurotoxicity and cardiovascular anomalies.

\section{Introduction}

Diabetes mellitus, a metabolic disorder, is associated with oxidative stress (Yan et al., 2014) as a result of reactive oxygen species (ROS) overproduction and reduction in antioxidant defense mechanisms (Ceretta et al., 2012; Fouda and AbdelRahman, 2017). The brain is more sensitive to oxidative stress, which affects gene expression and multiple cell functions (Giacco and Brownlee, 2010), due to its high oxygen consumption rate, plentiful lipid content, and relatively limited antioxidant mechanisms (Abdel Moneim, 2015). Similar to nitric oxide and heme oxygenase-derived carbon monoxide, hydrogen sulfide $\left(\mathrm{H}_{2} \mathrm{~S}\right)$ protects against diabetesinduced oxidative stress and cardiovascular complications (El-Sayed et al., 2016; van den Born et al., 2016); however, a mechanistic role for $\mathrm{H}_{2} \mathrm{~S}$ regulation in diabetes-evoked neurotoxicity remains unknown.

Dr. Mohamed Fouda is a visiting scholar from the Department of Pharmacology and Toxicology, Faculty of Pharmacy, Alexandria University, Egypt.

This work was supported by the National Institutes of Health [Grant 2R01 AA14441-10].

${ }^{1}$ Current affiliation: Zagazig University Faculty of Pharmacy, Zagazig, Egypt.

https://doi.org/10.1124/jpet.117.243865.
Gasotransmitters modulate the interaction between the central cardiovascular regulation and metabolic disorders such as diabetes mellitus (Szczepanska-Sadowska et al., 2010), and affect many brain regions such as the hippocampus, paraventricular nucleus, dorsal motor nucleus of the vagus, hypothalamus, and rostral ventrolateral medulla (RVLM) (Biessels et al., 2002; Szczepanska-Sadowska et al., 2010). The RVLM regulates sympathetic tone and blood pressure (Pilowsky and Goodchild, 2002; Madden and Sved, 2003), and RVLM oxidative stress increases sympathetic activity (Konno et al., 2012). While high glucoseevoked neuronal oxidative stress (Bahniwal et al., 2017) might contribute to the neurotoxicity, sympathoexcitation, and cardiovascular anomalies associated with diabetes, a definitive role for a dysfunctional cystathionine- $\gamma$ lyase $(\mathrm{CSE}) / \mathrm{H}_{2} \mathrm{~S}$ system in the RVLM of diabetic rats has not been investigated. Moreover, it should be noted that carbon monoxide and $\mathrm{H}_{2} \mathrm{~S}$-synthesizing enzymes colocalize in discrete brain areas (Ruginsk et al., 2015). Also, the $\mathrm{H}_{2} \mathrm{~S}$ dependent induction of heme oxygenase-1 (HO-1) in macrophages via the extracellular signal-regulated protein kinase 1 and 2 pathways (Oh et al., 2006) infers similar crosstalk in other cell types. It remains unknown if a deficit in the $\mathrm{H}_{2} \mathrm{~S} / \mathrm{HO}-1$ signaling underlies diabetes-induced neurotoxicity.

ABBREVIATIONS: CSE, cystathionine- $\gamma$ lyase; DCF, 2' ,7'-dichlorofluorescein; DCFH-DA, DCFH-DA; DHE, dihydroethidium; DLP, DL-propargylglycine; $\mathrm{HO}-1$, heme oxygenase- $1 ; \mathrm{H}_{2} \mathrm{~S}$, hydrogen sulfide; NaHS, sodium hydrogen sulfide; PBS, phosphate-buffered saline; ROS, reactive oxygen species; RVLM, rostral ventrolateral medulla; STZ, streptozotocin; TH, tyrosine hydoxylase. 
We have recently shown that moxonidine conferred cardioprotection by reversing the $\mathrm{CSE} / \mathrm{H}_{2} \mathrm{~S}$ dysfunction in the heart of diabetic rats (El-Sayed et al., 2016). Notably, moxonidine, a well-known centrally acting imidazoline $\mathrm{I}_{1}$ receptor agonist, improves cardiac function in hypertensive rats (MukaddamDaher et al., 2009). Also, $\mathrm{H}_{2} \mathrm{~S}$ modulates RVLM neuronal activity, which plays a vital role in hemodynamic control (Guo et al., 2011). Whether moxonidine protects RVLM neurons in diabetes and the mechanism of this neuroprotection have not been investigated.

The first objective of the current study was to ascertain a possible role for a deficit in $\mathrm{CSE} / \mathrm{H}_{2} \mathrm{~S}$ in diabetes-evoked RVLM neurotoxicity and sympathoexcitation. Afterward, we hypothesized that moxonidine mitigates the diabetes-induced RVLM neurotoxicity and sympathoexcitation by preserving neuronal $\mathrm{CSE} / \mathrm{H}_{2} \mathrm{~S} / \mathrm{HO}-1$ signaling.

\section{Materials and Methods}

Animals. Male Wistar rats (225-250 g; Charles River Laboratories, Raleigh, NC) were used and allowed free access to water and Purina chow (St. Louis, MO). Rats were retained on a balanced lightdark cycle and the temperature was kept at $22^{\circ} \mathrm{C} \pm 1^{\circ} \mathrm{C}$. All procedures were conducted in accordance with the Guide for the Care and Use of Laboratory Animals (2011) and approved by the institutional animal care and use committee https://www.pittsburgh. va.gov/Research/docs/Guide_8thEdition.pdf.

Experimental Groups. The brains used in the present study $(n=$ 5/group) were obtained from treated and untreated diabetic and control rats used in our recent study (El-Sayed et al., 2016). Briefly, male rats were fasted overnight (16 hours), treated with a freshly prepared single dose of streptozotocin (STZ) $(55 \mathrm{mg} / \mathrm{kg}$, i.p.) in $0.1 \mathrm{M}$ citrate buffer ( $\mathrm{pH} 4)$ or the buffer (control), and drinking water was substituted with 5\% dextrose for STZ-treated rats. Four weeks following diabetes induction (STZ injection), the rats were treated daily for 3 weeks with one of the following regimens or the vehicle (by the same route of administration): 1) $\mathrm{H}_{2} \mathrm{~S}$ donor, sodium hydrosulfide (NaHS) (3.4 mg/kg per day, i.p.); 2) CSE inhibitor, DL-propargylglycine (DLP) (37.5 mg/kg per day, i.p.); 3) moxonidine ( 2 or $6 \mathrm{mg} / \mathrm{kg}$ per day, gavage); and 4) a combination of moxonidine $(6 \mathrm{mg} / \mathrm{kg})$ and DLP. At the end of the cardiovascular studies (El-Sayed et al., 2016), 7 weeks after STZ injection, the animals were euthanized by overdose of pentobarbital, brains were collected, flash frozen in 2-methylbutane (Sigma-Aldrich, St Louis, MO) in dry ice, and stored at $-80^{\circ} \mathrm{C}$ until processed for the neurochemical studies detailed subsequently.

Neurochemical Studies. The RVLM was anatomically identified with the coordinates -12.6 to $-11.8 \mathrm{~mm}$ relative to bregma (Ibrahim and Abdel-Rahman, 2015), and used for the neurochemical measurements described subsequently. For histochemical measurements, three coronal sections containing the RVLM (14 $\mu \mathrm{m}$; approximately $0.05 \mathrm{~mm}$ ) were cut at $-24^{\circ} \mathrm{C}$ with a microtome cryostat (HM $505 \mathrm{E}$; Microm International $\mathrm{GmbH}$, Walldorf, Germany). The remaining RVLM tissue was collected with a $0.75 \mathrm{~mm}$ punch instrument (Stoelting Co., Wood Dale, IL), homogenized with phosphate-buffered saline (PBS) (for ROS measurement; $50 \mathrm{mM}$, pH 7.4) or with lysis buffer (for western blot analysis).

Quantification of Neurodegeneration (Fluorojade-C Staining). Modified protocols for immunofluorescence used in our previous studies (Wang and Abdel-Rahman, 2005) were used for staining degenerated neurons with a fluorescent Nissl counterstain (Yang et al., 2015). A fluorojade $\mathrm{C}$ staining kit was used in accordance with the manufacturer's instructions (TR-100-FJ; Biosensis, Thebarton, South Australia). Slides containing the brain sections were incubated in $0.06 \%$ potassium permanganate solution for 10 minutes followed by rinsing in distilled water for 2 minutes, and then incubated in fluorojade $\mathrm{C}$ solution (1:25) for 30 minutes. The slides were then washed and mounted on coverslips with Vecta-shield mounting medium (Vector Laboratories, Inc., Burlingame, CA). A Zeiss LSM 510 confocal microscope (Carl Zeiss, Inc., Thornwood, NY), and a blue (450-490 nm) excitation light was used for visualization of stained neurons and image acquisition (Yang et al., 2015). For quantification, the fluorescence intensity was measured in the RVLM using Zen Lite 2011 software (Carl Zeiss Microscopy GmbH, Germany) in brain sections from treatment and control groups ( $n=5$ brains/group).

RVLM Caspase-3 Expression. The immunohistochemistry technique described in our previous studies (Wang and Abdel-Rahman, 2002; Nassar et al., 2012) was used for measuring RVLM caspase-3 expression. Briefly, RVLM sections were postfixed for 2 hours in $4 \%$ paraformaldehyde in Tris-buffered saline, and subsequently incubated in $0.3 \% \mathrm{H}_{2} \mathrm{O}_{2}$ for 30 minutes to block endogenous peroxidase. Sections were then incubated with anti-caspase-3 polyclonal antibody (1:1000; Abcam, Cambridge, MA) overnight at $4^{\circ} \mathrm{C}$ using a modification of the avidin/biotin-complex method kit (Vector Laboratories, Inc.). For validation, control sections incubated only with the primary or secondary antibody showed no positive staining (data not shown). Neuronal profile counts, denoting the total number of caspase-3 immunoreactive neurons, were used for quantification of caspase-3 expression in an identical region (field $=0.125 \mathrm{~mm}^{2}$ ) of the RVLM of treated and control rats $(n=5)$. Positive profiles exhibited dark granular brown staining indicative of a 3,3-diaminobenzidine reaction product. The average per field count of positive neuronal profiles was then determined and subsequently converted into the number of profiles per unit area (square millimeter) for each rat brain (Marcus et al., 1998).

ROS Measurement. Oxidative stress was measured using $2^{\prime}, 7^{\prime}$-dichlorofluorescein diacetate (DCFH-DA), a general detector of oxidative species (Rezq and Abdel-Rahman, 2016; Fouda and AbdelRahman, 2017). Briefly, a stock solution of DCFH-DA (20 mM; Molecular Probes, Grand Island, NY) was prepared in methanol and kept at $-20^{\circ} \mathrm{C}$ protected from light. Punched RVLM tissues from treated and control groups were homogenized in PBS (50 mM, pH 7.4) and centrifuged at $14,000 \mathrm{rpm}$ for 20 minutes at $4^{\circ} \mathrm{C}$. Bio-Rad protein assay was used to quantify the proteins in the supernatant (Bio-Rad Laboratories, Hercules, CA). DCFH-DA stock solution was freshly diluted with PBS to prepare a $150 \mu \mathrm{M}$ working solution. The reaction was initiated by adding $10 \mu \mathrm{l}$ of RVLM homogenate supernatant in a 96-well plate to give a final concentration of $25 \mu \mathrm{M}$ DCFH-DA to produce fluorescent $2^{\prime}, 7^{\prime}$-dichlorofluorescein (DCF) in the incubation medium at $37^{\circ} \mathrm{C}$. Measurement of fluorescence intensity started 30 minutes later using a microplate fluorescence reader set at excitation $485 \mathrm{~nm} / \mathrm{emission} 530 \mathrm{~nm}$. The standard curve of DCF was constructed and the ROS level was determined as relative fluorescence units of generated DCF (Rezq and Abdel-Rahman, 2016).

Dihydroethidium (DHE) Staining for Superoxide Detection. Following the recent recommendations of utilizing two or more different methods for ROS level measurements (Griendling et al., 2016), frozen brain sections containing the RVLM from treated and control rats $(n=5)$ were incubated with $10 \mu \mathrm{M}$ DHE (Molecular Probes) at $37^{\circ} \mathrm{C}$ in the presence of $5 \% \mathrm{CO}_{2}$ in a moist chamber for 30 minutes. The assay was validated using positive and negative controls. A Zeiss LSM 510 microscope was used for image visualization (Carl Zeiss, Inc.). Image J Software (National Institutes of Health, Bethesda, MD) was used for quantification and the changes in total fluorescence intensity, normalized to control, were calculated (Collin et al., 2007).

Western Blot Analysis. The detection and quantification of the expression of CSE, HO-1, and tyrosine hydoxylase (TH) enzymes were followed as described in our previous studies (El-Sayed et al., 2016; Rezq and Abdel-Rahman, 2016; Fouda and Abdel-Rahman, 2017). Punched RVLM tissues were collected, as described previously, and homogenized with lysis buffer containing $20 \mathrm{mM}$ Tris, $\mathrm{Ph} 7.5,150 \mathrm{mM}$ $\mathrm{NaCl}, 1 \mathrm{mM}$ EDTA, $1 \mathrm{mM}$ EGTA, $1 \%$ Triton $\mathrm{x}-100,2.5 \mathrm{mM}$ sodium pyrophosphate, $1 \mathrm{mM} \beta$-glycerophosphate, and $1 \mu \mathrm{g} / \mathrm{ml}$ leuptin with 

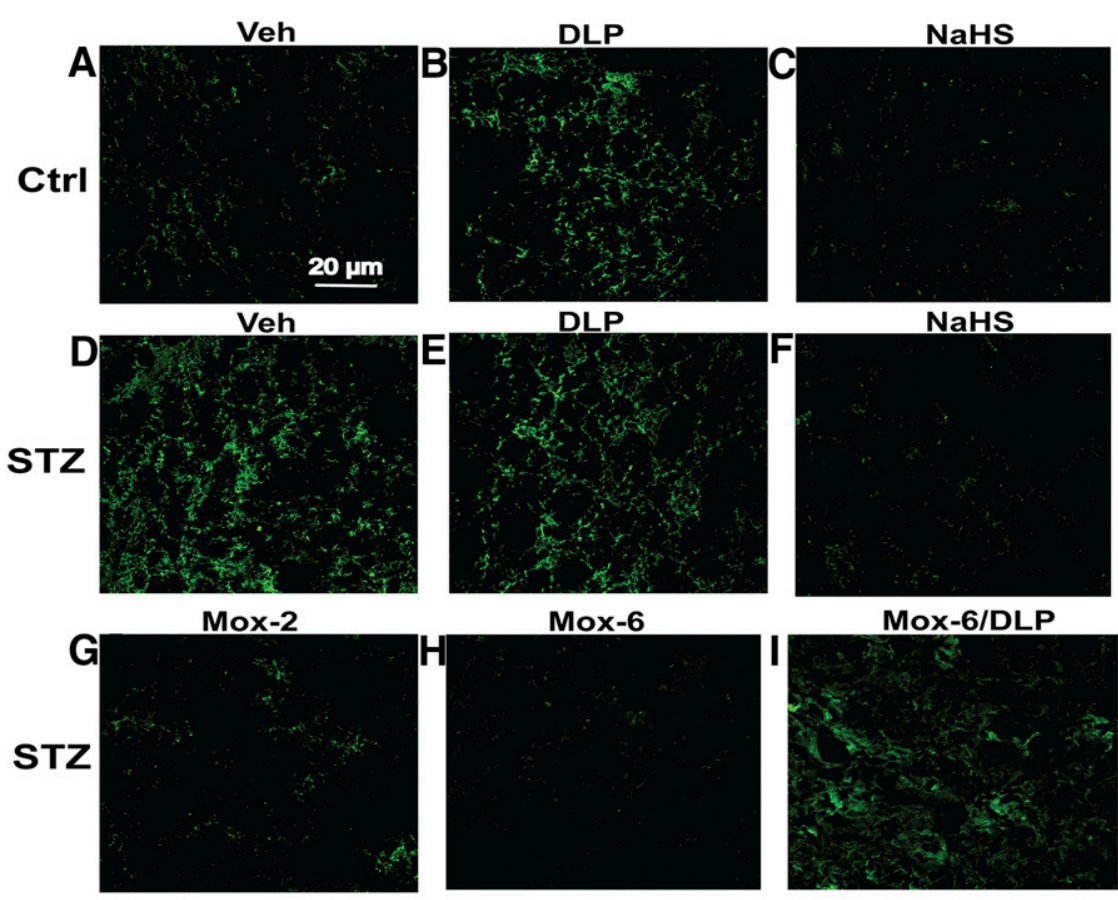

J

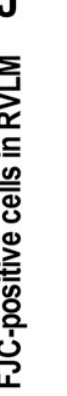

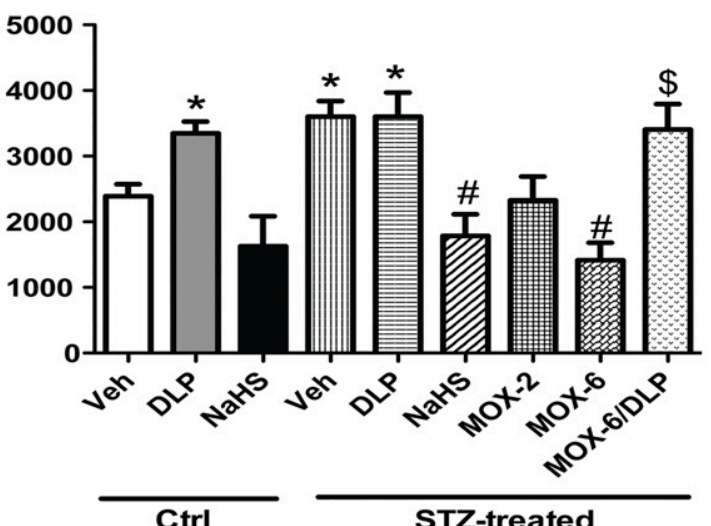

Fig. 1. FluoroJade C (FJC) positive cells examined in the RVLM of rats showing neurodegeneration. (A-I) Representative images of FJC-positive cells in male rats treated with STZ ( $55 \mathrm{mg} / \mathrm{kg}$, i.p. for 4 weeks) or its vehicle (buffer) receiving $\mathrm{NaHS}\left(\mathrm{H}_{2} \mathrm{~S}\right.$ donor, $3.4 \mathrm{mg} / \mathrm{kg}$ per day, i.p, for 3 weeks after diabetes induction), DLP (CSE inhibitor, $37.5 \mathrm{mg} / \mathrm{kg}$, i.p., for 3 weeks after diabetes induction), moxonidine (MOX) ( 2 or $6 \mathrm{mg} / \mathrm{kg}$ per day for 3 weeks after diabetes induction, gavage), a combination of MOX and DLP, or their vehicle (for 3 weeks after diabetes induction). (J) Group data showing the neurodegeneration expressed as the mean number of FJC-positive cells measured using National Institutes of Health ImageJ analysis of confocal images. Values are expressed as mean \pm S.E.M. $(n=5 \mathrm{rats} /$ group $) . * P<0.05$ vs corresponding control/vehicle (Ctrl/Veh) values; ${ }^{\#} P<$ 0.05 vs. corresponding STZ/Veh values; ${ }^{\$} P<0.05$ vs. STZ/MOX-6 values.

protease inhibitor cocktail (Roche Diagnostics, Indianapolis, IN). After homogenization and centrifugation, a Bio-Rad protein assay system (Bio-Rad Laboratories) was used to quantify proteins in the supernatant. Twenty micrograms of each protein were applied per lane of $4 \%-12 \%$ SDS-PAGE gel (Invitrogen, Carlsbad, CA) and transfer was done using nitrocellulose membranes. Then, the proteins were revealed by immunoblotting using a 1:500 dilution of anti-CSE, TH, or HO-1 polyclonal antibodies along with 1:5000 dilution of antiglyceraldehyde-3-phosphate dehydrogenase (for CSE and $\mathrm{TH}$ ) or anti- $\beta$-actin (for $\mathrm{HO}-1$ ) (Abcam) at $4^{\circ} \mathrm{C}$ overnight. Afterward, the membranes were washed and incubated for 60 minutes with mixture containing IRDye680-conjugated goat anti-mouse and IRDye800conjugated goat anti-rabbit (1:15000; LI-COR Biosciences, Lincoln, $\mathrm{NE}$ ). The identified proteins were visualized using an Odyssey Infrared Imager and analyzed with Odyssey application software version 5.2 (LI-COR Biosciences, Lincoln, NE). Data represent the mean values of the integrated density ratio of CSE, HO-1, or TH normalized to the corresponding housekeeping protein, glyceraldehyde3 -phosphate dehydrogenase, or $\beta$-actin, and expressed as percentage of nondiabetic control.

Measurement of RVLM $\mathrm{H}_{2} \mathrm{~S}$ Synthesizing Activity. The method described in our previous study (El-Sayed et al., 2016) was used. Punched RVLM tissues from different groups were homogenized in PBS (50 mM, pH 7.4), centrifuged, and then the protein in the supernatant was quantified using a Bio-Rad protein assay system (Bio-Rad Laboratories). We added $100 \mu \mathrm{l}$ sample (200 $\mu \mathrm{g}$ protein) to $900 \mu \mathrm{l}$ of the reaction mixture $(100 \mathrm{mM}$ potassium phosphate buffer $\mathrm{pH} 7.4,10 \mathrm{mM}$ L-cysteine, and $2 \mathrm{mM}$ pyridoxal 5'-phosphate). Cryovial tubes $(2 \mathrm{ml})$ containing $0.5 \mathrm{ml}$ of $1 \%$ zinc acetate and a filter paper $(1 \times 1.5 \mathrm{~cm})$ to increase the air-liquid contact were used to trap the released $\mathrm{H}_{2} \mathrm{~S}$ gas. The bottles were flushed with nitrogen and sealed with parafilm double layers. We started the reaction by incubating the bottles in a shaking water bath $\left(37^{\circ} \mathrm{C}\right)$ for 90 minutes. The reaction was stopped by adding $500 \mu \mathrm{l}$ of $50 \%$ trichloroacetic acid; the bottles were sealed again and returned to the shaking water bath for another 60 minutes to ensure trapping of all generated $\mathrm{H}_{2} \mathrm{~S}$. The contents were then transferred into Eppendorff tubes, and mixed with $134 \mu \mathrm{l}$ each of $N, N$-dimethyl-p-phenylene diamine sulfate $(20 \mathrm{mM})$ and ferric chloride $(30 \mathrm{mM})$ followed by 20 -minute incubation at room temperature. Finally, the contents were transferred into a 96 -well plate and read at $650 \mathrm{~nm}$ in a microplate reader. We calculated the $\mathrm{H}_{2} \mathrm{~S}$ concentrations using a calibration curve constructed with $\mathrm{NaHS}$ solution in $50 \mathrm{mM}$ potassium phosphate buffer, pH $6.8(0-320 \mu \mathrm{M}$ NaHS equivalent to $0-96 \mu \mathrm{M} \mathrm{H}_{2} \mathrm{~S}$ ). The $\mathrm{H}_{2} \mathrm{~S}$ concentration was 

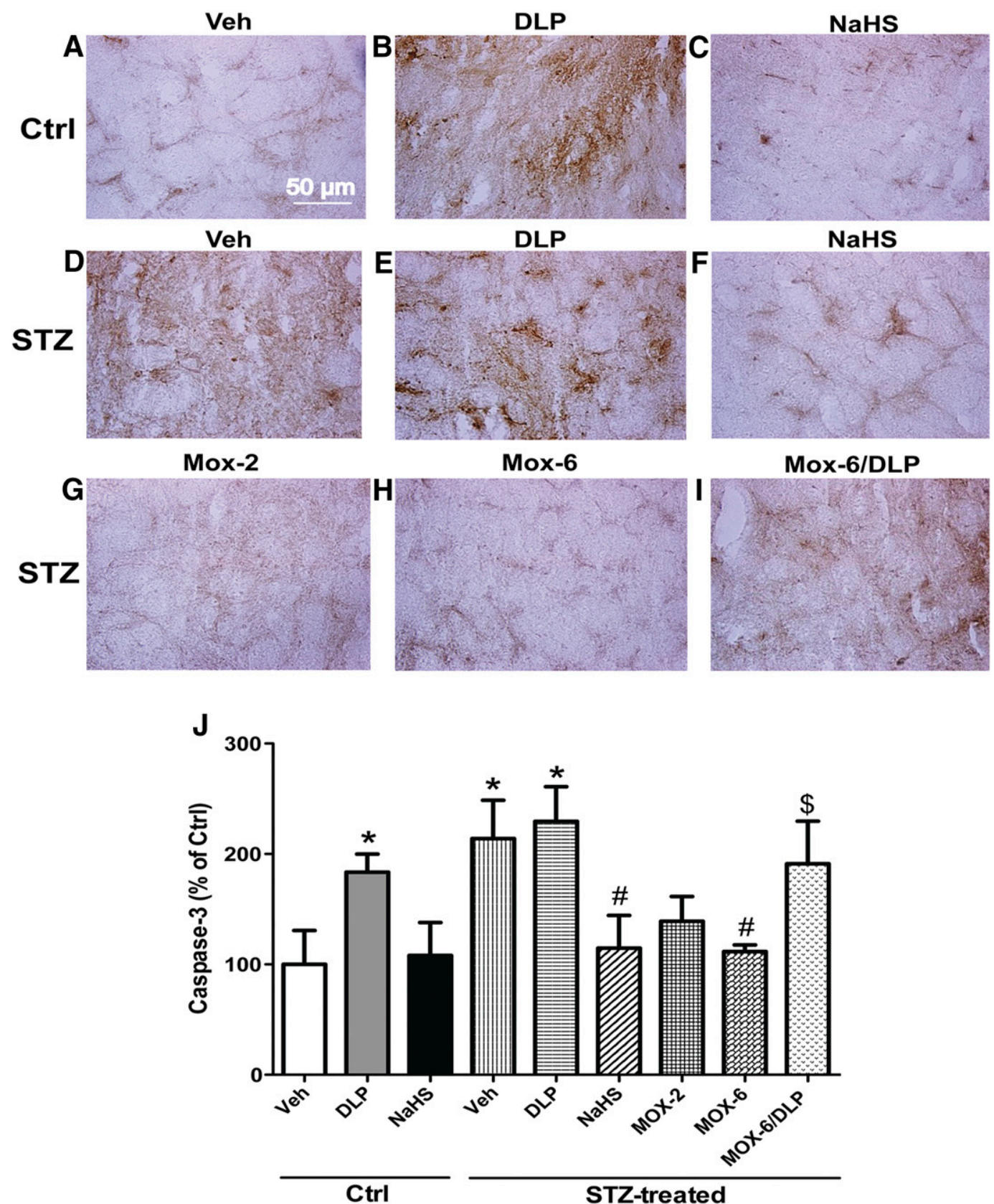

Fig. 2. Immunohistochemical detection of caspase-3 examined in the RVLM of rats. (A-I) Representative images of caspase-3 expression in male rats treated with STZ ( $55 \mathrm{mg} / \mathrm{kg}$, i.p., for 4 weeks) or its vehicle (buffer) receiving $\mathrm{NaHS}\left(\mathrm{H}_{2} \mathrm{~S}\right.$ donor for 3 weeks after diabetes induction; $3.4 \mathrm{mg} / \mathrm{kg}$ per day), DLP (CSE inhibitor, $37.5 \mathrm{mg} / \mathrm{kg}$, i.p., for 3 weeks after diabetes induction), moxonidine (MOX) ( 2 or $6 \mathrm{mg} / \mathrm{kg}$ per day for 3 weeks after diabetes induction, gavage), a combination of MOX and DLP, or their vehicle (for 3 weeks after diabetes induction). (J) Group data showing the mean number of caspase-3 expression measured using National Institutes of Health ImageJ analysis of confocal images. Values are expressed as mean \pm S.E.M. $(n=5$ rats/group). ${ }^{*} P<0.05$ vs. corresponding control/vehicle $(\mathrm{Ctrl} / \mathrm{Veh})$ values; ${ }^{\#} P<0.05$ vs. corresponding STZ/Veh values; ${ }^{\$} P<0.05$ vs. STZ/MOX-6 values.

calculated as $30 \%$ of the NaHS concentration as previously reported (Velasco-Xolalpa et al., 2013; El-Sayed et al., 2016), and the RVLM $\mathrm{H}_{2} \mathrm{~S}$ enzyme-synthesizing activity was expressed as nanomoles per milligrams protein per minutes.

Drugs. The following drugs and chemicals were used in the present study. Moxonidine (American Custom Chemicals Corp., San Diego, CA), DLP (Chem-Impex International Inc., Wood Dale, IL), $N, N$-dimethyl-pphenylenediamine sulfate (Acros Organics Thermo Fisher Scientific, Bridgewater, NJ), and acrylamide 40\% (Fischer Scientific, Pittsburgh, PA). All other chemicals were purchased from Sigma-Aldrich.

Data Analysis and Statistics. Data are expressed as mean \pm S.E. M. Statistical analyses were conducted by one-way or repeated measures analysis of variance for multiple comparisons followed by Tukey's post hoc test and Student's $t$ test using Prism 5.0 software (GraphPad Software Inc., San Diego, CA); $P<0.05$ was considered significant.

\section{Results}

Moxonidine Mitigates STZ-Induced RVLM Neurodegeneration and Oxidative Stress. Fluorojade C staining, indicative of neuronal injury (Chaparro et al., 2013), was used to determine the number of RVLM-damaged neurons. The RVLM of STZ-diabetic rats or DLP (CSE inhibitor)-treated 
nondiabetic rats exhibited approximately 2 -fold higher number of damaged neurons compared with the nondiabetic (vehicletreated) control group (Fig. 1). Moxonidine (dose dependantly) or NaHS $\left(\mathrm{H}_{2} \mathrm{~S}\right.$ donor) reduced $(P<0.05)$ the number of RVLMdamaged neurons in STZ-diabetic rats, and DLP abolished the neuroprotective effect of moxonidine $(6 \mathrm{mg} / \mathrm{kg})$ in STZ-treated rats (Fig. 1).

Similar to the fluorojade findings, the number of RVLM caspase-3 immunoreactive neurons was higher $(P<0.05)$ in STZ-diabetic and DLP-treated nondiabetic rats compared with the nondiabetic control group (Fig. 2). Moxonidine (dose dependently) or NaHS $\left(\mathrm{H}_{2} \mathrm{~S}\right.$ donor $)$ reduced $(P<0.05)$ the number of RVLM caspase-3 immunoreactive neurons in STZtreated rats, and the neuroprotective effect of moxonidine $(6 \mathrm{mg} / \mathrm{kg}$ ) in STZ-treated rats was diminished by DLP (CSE inhibitor) coadministration (Fig. 2).

DCF kinetics (Fig. 3) and DHE fluorescence staining intensity (Fig. 4) showed that STZ-diabetic rats or DLP-treated nondiabetic rats exhibited higher $(P<0.05)$ ROS levels compared with the nondiabetic control group. NaHS or moxonidine reversed the increase in ROS level in STZdiabetic rats (Figs. 3 and 4), and DLP diminished the favorable effect of moxonidine $(6 \mathrm{mg} / \mathrm{kg})$ on RVLM redox status in STZdiabetic rats (Figs. 3 and 4). Furthermore, NaHS attenuated the increased ROS level and neuronal damage in STZ-treated rats, but it had no effect in control rats (Figs. 1-4).

Moxonidine or NaHS Restores CSE, HO-1, and TH in the RVLM of Diabetic Rats. Western blot analysis showed an increase $(P<0.05)$ in TH (Fig. 5A) and reductions $(P<$ 0.05) in CSE (Fig. 5B) and HO-1 (Fig. 5C) expressions in the RVLM of STZ-diabetic rats compared with nondiabetic control rats. NaHS or moxonidine reversed these STZ-evoked effects

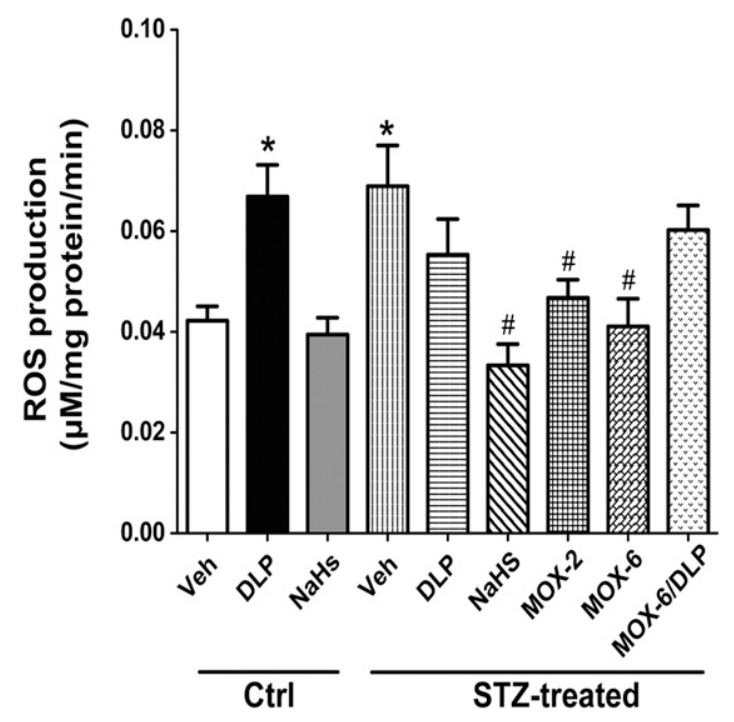

Fig. 3. The DCF biochemical assay of the generation of ROS showing the slopes (regression coefficients) of the regression lines representing the rate of ROS production in the RVLM of male rats treated with STZ (55 mg/kg, i.p., for 4 weeks) or its vehicle (buffer) receiving $\mathrm{NaHS}\left(\mathrm{H}_{2} \mathrm{~S}\right.$ donor for 3 weeks after diabetes induction; $3.4 \mathrm{mg} / \mathrm{kg}$ per day), DLP (CSE inhibitor, $37.5 \mathrm{mg} / \mathrm{kg}$, i.p., for 3 weeks after diabetes induction), moxonidine (MOX) ( 2 or $6 \mathrm{mg} / \mathrm{kg}$ per day for 3 weeks after diabetes induction, gavage), a combination of MOX and DLP, or their vehicle (for 3 weeks after diabetes induction). Values are expressed as mean \pm S.E.M. ( $n=5$ rats/group). $* P<0.05$ vs. corresponding control/vehicle $(\mathrm{Ctrl} / \mathrm{Veh})$ values; ${ }^{*} P<0.05$ vs. corresponding STZ/Veh values. and restored the protein levels of these enzymes to nondiabetic control levels (Fig. 5). Except for DLP-evoked reduction $(P<0.05)$ in CSE, NaHS or DLP had no effect on the expression level of these proteins in the RVLM of nondiabetic control rats (Fig. 5). However, DLP coadministration prevented the restoration of RVLM CSE, HO-1, and TH levels caused by moxonidine $(6 \mathrm{mg} / \mathrm{kg})$ in STZ-diabetic rats (Fig. 5). Finally, CSE activity was substantially $(P<0.05)$ reduced in the RVLM of STZ-diabetic rats and DLP-treated nondiabetic rats (Fig. 6). NaHS or moxonidine $(6 \mathrm{mg} / \mathrm{kg})$ reversed the reduction in CSE activity in the RVLM of STZ-diabetic rats, and concurrent DLP administration prevented the favorable effect of moxonidine on RVLM CSE activity (Fig. 6).

\section{Discussion}

The present study is the first to discern a physiologic neuroprotective role for $\mathrm{H}_{2} \mathrm{~S}$ in a major cardiovascular regulating nucleus, the RVLM. Our findings also suggest $\mathrm{H}_{2} \mathrm{~S}$-dependent neuroprotective effect for moxonidine ( $\mathrm{I}_{1}$ agonist) against diabetes-induced RVLM neuronal injury, oxidative stress, and sympathoexcitation. The main findings that support our conclusions are the following: 1) NaHS $\left(\mathrm{H}_{2} \mathrm{~S}\right.$ donor) or moxonidine mitigated the diabetes-induced RVLM neuronal injury, apoptosis, and oxidative stresslinked sympathoexcitation; 2) either intervention reversed the diabetes-induced reductions in CSE activity and in CSE and HO-1 expressions in the RVLM; and 3) CSE inhibition (DLP) reproduced a diabetic RVLM phentotype in nondiabetic rats and nullified the favorable RVLM neuroprotective effects of moxonidine. Together, these findings implicate $\mathrm{CSE} / \mathrm{H}_{2} \mathrm{~S}$ in moxonidine-evoked alleviation of diabetesevoked neurotoxicity.

Our recent study raised important questions about the mechanism of the sympathoexcitation, which was associated with hypertension and autonomic dysregulation in diabetic rats (El-Sayed et al., 2016). Here, we addressed this question by testing the hypothesis that RVLM oxidative stress/ neurotoxicity plays a pivotal role in these diabetes-evoked effects for the following reasons. First, the impaired glycemic control associated with diabetes activates RVLM neurons (Oshima et al., 2017), although this evidence was obtained in vitro and the mechanisms of this effect remain unknown. Second, whether the inhibition of CSE-derived $\mathrm{H}_{2} \mathrm{~S}$, which contributes to diabetes-evoked cardiac and autonomic dysfunction in vivo (El-Sayed et al., 2016), occurs and accounts for similar effects in the RVLM has not been investigated. To address these questions, we conducted detailed studies on the RVLM tissues obtained from diabetic and control rats used in our recent study (El-Sayed et al., 2016).

As an important foundation, our current study showed that STZ-diabetic rats exhibited RVLM injury as indicated by the number of degenerated neurons identified by fluorojade $\mathrm{C}$ staining (Fig. 1), and by increased neuronal apoptosis (Fig. 2). While this new finding replicates neurotoxicity in other brain nuclei of the same model (Wang et al., 2014), the mechanism of such neurotoxicity has not been investigated.

We focused on neuronal oxidative stress as an underlying mechanism for the diabetes-evoked neuronal injury and sympathoexcitation based on current evidence in different model systems (Wang et al., 2014; Fouda and Abdel-Rahman, 2017; Oshima et al., 2017). In accordance with current guidelines 

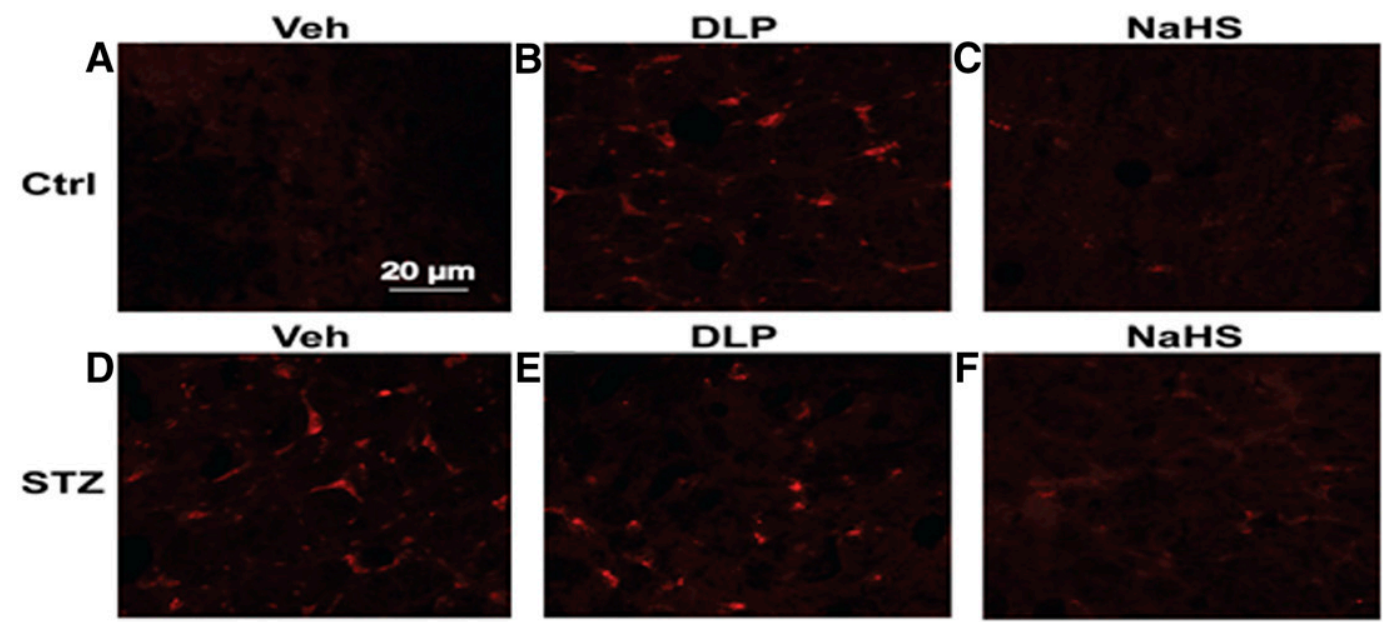

DLP

NaHS
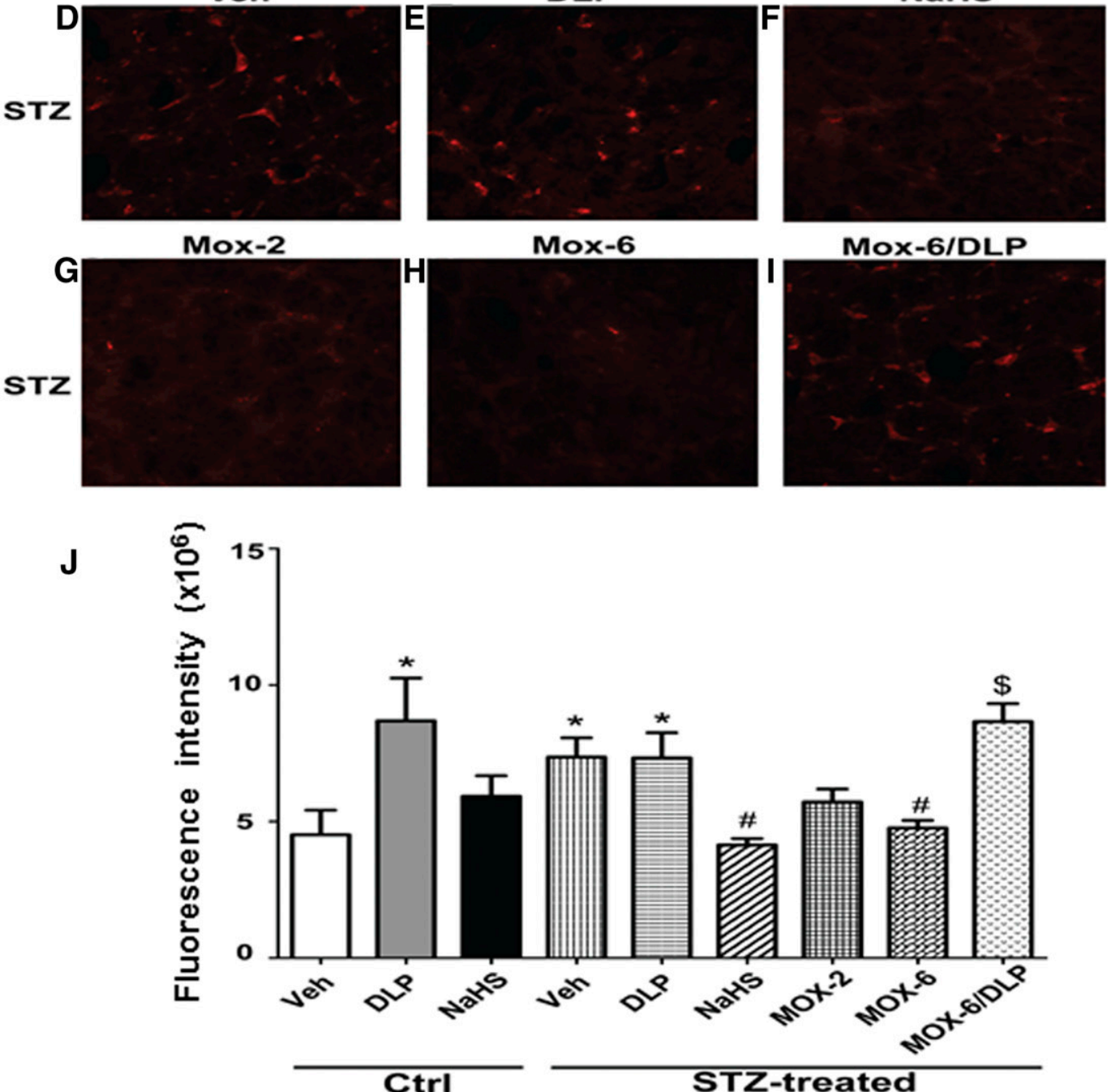

Fig. 4. (A-I) Confocal images showing superoxide level indicated by DHE staining (red) in the RVLM of male rats treated with STZ (55 mg/kg, i.p., for 4 weeks) or its vehicle (buffer) receiving NaHS $\left(\mathrm{H}_{2} \mathrm{~S}\right.$ donor for 3 weeks after diabetes induction; $3.4 \mathrm{mg} / \mathrm{kg}$ per day), DLP (CSE inhibitor, $37.5 \mathrm{mg} / \mathrm{kg}$, i.p., for 3 weeks after diabetes induction), moxonidine (MOX) (2 or $6 \mathrm{mg} / \mathrm{kg}$ per day for 3 weeks after diabetes induction; gavage), a combination of MOX and DLP, or their vehicle (for 3 weeks after diabetes induction). (J) Values are expressed as mean \pm S.E.M. $\left(n=5\right.$ rats/group). ${ }^{*} P<0.05$ vs. corresponding control/vehicle (Ctrl/Veh) values; ${ }^{\#} P<0.05$ vs. corresponding STZ/Veh values; ${ }^{\$} P<0.05$ vs. STZ/MOX- 6 values.

(Griendling et al., 2016), we confirmed the diabetes-evoked increase in RVLM ROS by two different assays (DCF and DHE). Evidence suggests that the diabetes-evoked neuronal oxidative stress, observed here (Figs. 3 and 4) and in reported studies, could be caused by glucose autoxidation, endoplasmic reticulum stress, and impaired antioxidant defenses ( $\mathrm{Li}$ et al., 2005; Correia et al., 2008) as well as the increased vulnerability of the brain to oxidative stress (Carvalho et al., 2012; Duarte et al., 2013).

The results of the present study and reported findings suggest a causal role for local oxidative stress in the diabetes-evoked sympathoexcitation (increased $\mathrm{TH}$, see Fig. 5A) in the RVLM. Notably, TH in the RVLM reflects sympathetic activity (Guyenet, 2006) and oxidative stress induces sympathoexcitation in brain stem nuclei (Zimmerman and Davisson, 2004; Huang et al., 2006; Fujita et al., 2012). Furthermore, sympathoexcitation exacerbates neurodegeneration (Burke et al., 2004) and may contribute to cardiovascular anomalies in the same STZ-diabetic rats (El-Sayed et al., 2016) because the RVLM serves a pivotal role in blood pressure regulation (Pilowsky and Goodchild, 2002; Madden and Sved, 2003). 

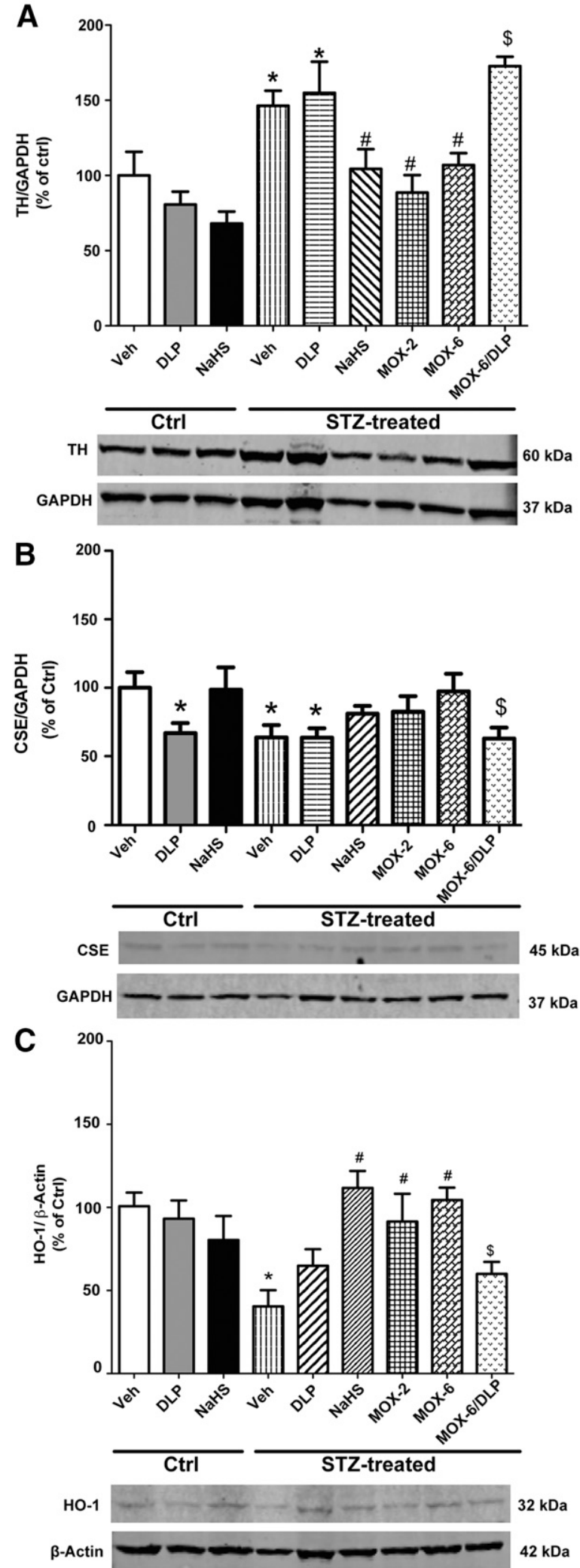

Fig. 5. Western blot analyses showing the protein expression in the RVLM of male rats treated with STZ ( $55 \mathrm{mg} / \mathrm{kg}$, i.p., for 4 weeks) or its vehicle (buffer) receiving NaHS $\left(\mathrm{H}_{2} \mathrm{~S}\right.$ donor for 3 weeks after diabetes induction; $3.4 \mathrm{mg} / \mathrm{kg}$ per day), DLP (CSE inhibitor; $37.5 \mathrm{mg} / \mathrm{kg}$, i.p., for 3 weeks after diabetes induction), moxonidine (MOX) (2 or $6 \mathrm{mg} / \mathrm{kg}$ per day for 3 weeks after diabetes induction, gavage), a combination of MOX and $\mathrm{DLP}$, or their vehicle (for 3 weeks after diabetes induction). (A) $\mathrm{TH}$ ratio to glyceraldehyde-3-phosphate dehydrogenase (GAPDH) protein (housekeeping
The present findings suggest a pivotal role for $\mathrm{CSE} / \mathrm{H}_{2} \mathrm{~S}$ downregulation (Fig. 5B) in diabetes-induced oxidative stress and the subsequent RVLM neurotoxicity (Figs. 1-5) given the antioxidant and anti-inflammatory actions of $\mathrm{H}_{2} \mathrm{~S}$ (Mustafa et al., 2009). This premise is supported by the ability of CSE inhibition (DLP) to cause oxidative stress and to reproduce the diabetic phenotype in the RVLM of nondiabetic rats. Notably, the new finding that DLP reduced CSE protein levels in these nondiabetic rats (Fig. 5B) likely resulted from DLP-evoked oxidative stress (Figs. 3 and 4) via the inhibition of CSE catalytic activity (Fig. 6). This possibility is supported by the finding that $\mathrm{H}_{2} \mathrm{O}_{2}$-evoked oxidative stress suppressed CSE protein level in cultured cells (Manna et al., 2014), and by the inverse relationship between ROS and CSE expression in the RVLM (Figs. 3-5) and liver (Manna et al., 2014) of STZdiabetic rats. These findings suggest an inhibitory role for oxidative stress on CSE protein expression, and identify CSE/ $\mathrm{H}_{2} \mathrm{~S}$ upregulation as a novel target for the alleviation of RVLM neurotoxicity in diabetes.

The results of the present study show that moxonidine inhibits sympathoexcitaion (Fig. 5A) and neuronal death (Figs. 1 and 2) in diabetic rats. These findings agree with neuroprotective effects of moxonidine against ischemic insults in neuronal cultures (Milhaud et al., 2000; Bakuridze et al., 2009) and against glutamate-evoked neurotoxicity (Keller and García-Sevilla, 2016). However, the mechanism of the neuroprotective effect of moxonidine was not investigated in the reported studies.

Our findings suggest a pivotal role for RVLM CSE/ $\mathrm{H}_{2} \mathrm{~S}$ upregulation in moxonidine-evoked neuroprotection and sympathoinhibition because these responses were tightly correlated in moxonidine-treated diabetic rats and were abolished in the presence of CSE inhibition (DLP). These findings are consistent with the neuroprotective effect of $\mathrm{H}_{2} \mathrm{~S}$ and its antiapoptotic effect through increasing glutathione level and suppressing oxidative stress (Kimura and Kimura, 2004; Kimura et al., 2010; Mikami et al., 2016). These reported findings raised the possibility that $\mathrm{H}_{2} \mathrm{~S}$ interacts with another antioxidant gaseous neuromodulator, HO-1.

We studied the role of $\mathrm{HO}-1$ in our model system because it is expressed in the RVLM neurons (Mazza et al., 2001), exerts neuronal antioxidant and antiapoptotic effects (Spitz et al., 1987; Fouda and Abdel-Rahman, 2017; Kim et al., 2017), and mediates sympathoinhibition (Nassar et al., 2011). Our findings suggest $\mathrm{H}_{2} \mathrm{~S}$-dependent regulation of HO-1 in the RVLM contributes to the diabetes-evoked neurotoxicity and its alleviation by moxonidine for the following reasons. First, $\mathrm{CSE} / \mathrm{H}_{2} \mathrm{~S}$ inhibition in diabetic and healthy rats following DLP was associated with reduced RVLM HO-1 expression (Fig. 5C). Second, the $\mathrm{H}_{2} \mathrm{~S}$ donor NaHS or moxonidine restored RVLM HO-1 expression in diabetic rats (Fig. 5C). Third, CSE inhibition (DLP) abolished the moxonidine-evoked restoration of HO-1 expression in diabetic rats (Fig. 5C).

protein) and western bands depicting the protein expression are shown below the bar graphs. (B) CSE ratio to GAPDH protein (housekeeping protein) and western bands depicting the protein expression are shown below the bar graphs. (C) HO-1 ratio to $\beta$-actin protein (housekeeping protein) and western bands depicting the protein expression are shown below the bar graphs. Values are expressed as mean \pm S.E.M. ( $n=5$ rats/group). $* P<0.05$ vs. corresponding control/vehicle $(\mathrm{Ctrl} / \mathrm{Veh})$ values; ${ }^{\#} P<0.05$ vs. corresponding STZ/Veh values; ${ }^{\$} P<0.05$ vs. STZ/MOX-6 values. 


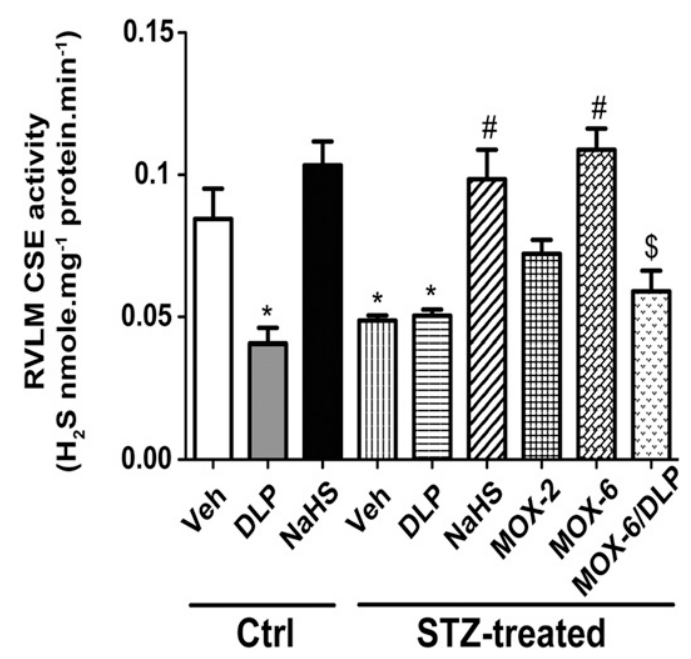

Fig. 6. $\mathrm{H}_{2} \mathrm{~S}$ synthesizing enzyme activity in the RVLM of male rats treated with STZ $(55 \mathrm{mg} / \mathrm{kg}$, i.p., for 4 weeks) or its vehicle (buffer) receiving $\mathrm{NaHS}\left(\mathrm{H}_{2} \mathrm{~S}\right.$ donor for 3 weeks after diabetes induction; $3.4 \mathrm{mg} / \mathrm{kg}$ per day), DLP (CSE inhibitor, $37.5 \mathrm{mg} / \mathrm{kg}$, i.p., for 3 weeks after diabetes induction), moxonidine (MOX) (2 or $6 \mathrm{mg} / \mathrm{kg}$ per day for 3 weeks after diabetes induction, gavage), a combination of MOX and DLP, or their vehicle (for 3 weeks after diabetes induction). Values are expressed as mean \pm S.E.M. ( $n=5$ rats/group). ${ }^{*} P<0.05$ vs. corresponding control vehicle $(\mathrm{Ctrl} / \mathrm{Veh})$ values; ${ }^{\#} P<0.05$ vs. corresponding $\mathrm{STZ} / \mathrm{Veh}$ values; ${ }_{P}<0.05$ vs. STZ/MOX- 6 values.

The present findings provide two new pieces of evidence. First, $\mathrm{CSE} / \mathrm{H}_{2} \mathrm{~S}$ inhibition mediates neuronal injury, oxidative stress, and increased presympathetic neuronal activity in the RVLM in diabetic rats. Second, restoration of RVLM CSE-derived $\mathrm{H}_{2} \mathrm{~S}$ mediates the sympathoinhibitory and neuroprotective actions of moxonidine in diabetes. The neuropathological consequences of diabetes and their reversal by moxonidine might explain the cardiovascular anomalies and their alleviation by moxonidine, respectively, in our previous in vivo study (El-Sayed et al., 2016). The findings also suggest that $\mathrm{H}_{2} \mathrm{~S}$ confers neuroprotection and sympathoinhibition, at least partly, via HO-1, and highlight the RVLM CSE/HO-1 pathway as a viable target for developing novel therapeutics for alleviating the neurotoxicity and cardiovascular anomalies associated with diabetes.

\section{Acknowledgments}

We thank Kui Sun and Dr. Fanrong Yao for technical assistance.

\section{Authorship Contributions}

Participated in research design: Fouda, El-Sayed, Abdel-Rahman. Conducted experiments: Fouda.

Performed data analysis: Fouda.

Wrote or contributed to the writing of the manuscript: Fouda, Abdel-Rahman.

\section{References}

Abdel Moneim AE (2015) The neuroprotective effect of berberine in mercury-induced neurotoxicity in rats. Metab Brain Dis 30:935-942.

Bahniwal M, Little JP, and Klegeris A (2017) High glucose enhances neurotoxicity and inflammatory cytokine secretion by stimulated human astrocytes. Curr Alzheimer Res 14:731-741.

Bakuridze K, Savli E, Gongadze N, Baş DB, and Gepdiremen A (2009) Protection in glutamate-induced neurotoxicity by imidazoline receptor agonist moxonidine. Int $J$ Neurosci 119:1705-1717.

Biessels GJ, van der Heide LP, Kamal A, Bleys RL, and Gispen WH (2002) Ageing and diabetes: implications for brain function. Eur $J$ Pharmacol 441:1-14.

Burke WJ, Li SW, Chung HD, Ruggiero DA, Kristal BS, Johnson EM, Lampe P, Kumar VB, Franko M, Williams EA, et al. (2004) Neurotoxicity of MAO metabolites of catecholamine neurotransmitters: role in neurodegenerative diseases. Neurotoxicology 25:101-115.
Carvalho C, Cardoso S, Correia SC, Santos RX, Santos MS, Baldeiras I, Oliveira CR, and Moreira PI (2012) Metabolic alterations induced by sucrose intake and Alzheimer's disease promote similar brain mitochondrial abnormalities. Diabetes 61: 1234-1242.

Ceretta LB, Réus GZ, Abelaira HM, Ribeiro KF, Zappellini G, Felisbino FF, Steckert AV, Dal-Pizzol F, and Quevedo J (2012) Increased oxidative stress and imbalance in antioxidant enzymes in the brains of alloxan-induced diabetic rats. Exp Diabetes Res 2012:302682.

Chaparro RE, Quiroga C, Bosco G, Erasso D, Rubini A, Mangar D, Parmagnani A, and Camporesi EM (2013) Hippocampal cellular loss after brief hypotension. Springerplus 2:23.

Collin B, Busseuil D, Zeller M, Perrin C, Barthez O, Duvillard L, Vergely C, Bardou M, Dumas M, Cottin Y, et al. (2007) Increased superoxide anion production is associated with early atherosclerosis and cardiovascular dysfunctions in a rabbit model. Mol Cell Biochem 294:225-235.

Correia S, Carvalho C, Santos MS, Seiça R, Oliveira CR, and Moreira PI (2008) Mechanisms of action of metformin in type 2 diabetes and associated complications: an overview. Mini Rev Med Chem 8:1343-1354.

Duarte AI, Candeias E, Correia SC, Santos RX, Carvalho C, Cardoso S, Plácido A, Santos MS, Oliveira CR, and Moreira PI (2013) Crosstalk between diabetes and brain: glucagon-like peptide-1 mimetics as a promising therapy against neurodegeneration. Biochim Biophys Acta 1832:527-541.

El-Sayed SS, Zakaria MN, Abdel-Ghany RH, and Abdel-Rahman AA (2016) Cystathionine- $\gamma$ lyase-derived hydrogen sulfide mediates the cardiovascular protective effects of moxonidine in diabetic rats. Eur J Pharmacol 783:73-84.

Fouda MA and Abdel-Rahman AA (2017) Endothelin confers protection against high glucose-induced neurotoxicity via alleviation of oxidative stress. $J$ Pharmacol Exp Ther 361:130-139.

Fujita M, Ando K, Kawarazaki H, Kawarasaki C, Muraoka K, Ohtsu H, Shimizu H, and Fujita T (2012) Sympathoexcitation by brain oxidative stress mediates arterial pressure elevation in salt-induced chronic kidney disease. Hypertension 59:105-112.

Giacco F and Brownlee M (2010) Oxidative stress and diabetic complications. Circ Res 107:1058-1070.

Griendling KK, Touyz RM, Zweier JL, Dikalov S, Chilian W, Chen YR, Harrison DG, and Bhatnagar A; American Heart Association Council on Basic Cardiovascular Sciences (2016) Measurement of reactive oxygen species, reactive nitrogen species, and redox-dependent signaling in the cardiovascular system: a scientific statement from the American Heart Association. Circ Res 119:e39-e75.

Guo Q, Jin S, Wang XL, Wang R, Xiao L, He RR, and Wu YM (2011) Hydrogen sulfide in the rostral ventrolateral medulla inhibits sympathetic vasomotor tone through ATP-sensitive $\mathrm{K}^{+}$channels. J Pharmacol Exp Ther 338:458-465.

Guyenet PG (2006) The sympathetic control of blood pressure. Nat Rev Neurosci 7: $335-346$.

Huang BS, Amin MS, and Leenen FH (2006) The central role of the brain in saltsensitive hypertension. Curr Opin Cardiol 21:295-304.

Ibrahim BM and Abdel-Rahman AA (2015) A pivotal role for enhanced brainstem Orexin receptor 1 signaling in the central cannabinoid receptor 1-mediated pressor response in conscious rats. Brain Res 1622:51-63.

Keller B and García-Sevilla JA (2016) Inhibitory effects of imidazoline receptor ligands on basal and kainic acid-induced neurotoxic signalling in mice. J Psychopharmacol 30:875-886.

Kim S, Chin YW, and Cho J (2017) Protection of cultured cortical neurons by luteolin against oxidative damage through inhibition of apoptosis and induction of heme oxygenase-1. Biol Pharm Bull 40:256-265.

Kimura Y, Goto Y, and Kimura H (2010) Hydrogen sulfide increases glutathione production and suppresses oxidative stress in mitochondria. Antioxid Redox Signal 12:1-13.

Kimura Y and Kimura H (2004) Hydrogen sulfide protects neurons from oxidative stress. FASEB J 18:1165-1167.

Konno S, Hirooka Y, Kishi T, and Sunagawa K (2012) Sympathoinhibitory effects of telmisartan through the reduction of oxidative stress in the rostral ventrolateral medulla of obesity-induced hypertensive rats. J Hypertens 30:1992-1999.

Li ZG, Zhang W, and Sima AA (2005) The role of impaired insulin/IGF action in primary diabetic encephalopathy. Brain Res 1037:12-24.

Madden CJ and Sved AF (2003) Rostral ventrolateral medulla C1 neurons and cardiovascular regulation. Cell Mol Neurobiol 23:739-749.

Manna P, Gungor N, McVie R, and Jain SK (2014) Decreased cystathionine- $\gamma$-lyase (CSE) activity in livers of type 1 diabetic rats and peripheral blood mononuclear cells (PBMC) of type 1 diabetic patients. J Biol Chem 289:11767-11778.

Marcus DL, Strafaci JA, Miller DC, Masia S, Thomas CG, Rosman J, Hussain S, and Freedman ML (1998) Quantitative neuronal c-Fos and c-Jun expression in Alzheimer's disease. Neurobiol Aging 19:393-400.

Mazza E, Thakkar-Varia S, Tozzi CA, and Neubauer JA (2001) Expression of heme oxygenase in the oxygen-sensing regions of the rostral ventrolateral medulla. $J$ Appl Physiol (1985) 91:379-385.

Mikami Y, Kakizawa S, and Yamazawa T (2016) Essential roles of natural products and gaseous mediators on neuronal cell death or survival. Int J Mol Sci 17:1652.

Milhaud D, Fagni L, Bockaert J, and Lafon-Cazal M (2000) Imidazoline-induced neuroprotective effects result from blockade of NMDA receptor channels in neuronal cultures. Neuropharmacology 39:2244-2254.

Mukaddam-Daher S, Menaouar A, Paquette PA, Jankowski M, Gutkowska J, Gillis MA, Shi YF, Calderone A, and Tardif JC (2009) Hemodynamic and cardiac effects of chronic eprosartan and moxonidine therapy in stroke-prone spontaneously hypertensive rats. Hypertension 53:775-781.

Mustafa AK, Gadalla MM, and Snyder SH (2009) Signaling by gasotransmitters. Sci Signal 2:re2.

Nassar NN, Abdelsalam RM, Abdel-Rahman AA, and Abdallah DM (2012) Possible involvement of oxidative stress and inflammatory mediators in the protective effects of the early preconditioning window against transient global ischemia in rats. Neurochem Res 37:614-621. 
178

Fonda et al.

Vassar NN, Li G, Strut AL, and Abdel-Rahman AA (2011) Enhanced hemeoxygenase activity in the rostral ventrolateral medulla mediates exaggerated hemin-evoked hypotension in the spontaneously hypertensive rat. J Pharmacol Exp Then 339:267-274.

Oh GS, Pe HO, Lee BS, Kim BN, Kim JM, Kim HR, Jon SB, Jon WK, Cha HJ, and Chung HT (2006) Hydrogen sulfide inhibits nitric oxide production and nuclear factor- $\kappa$ B via heme oxygenase-1 expression in RAW 264.7 macrophages stimulated with lipopolysaccharide. Free Radic Biol Med 41:106-119.

Oshima N, Onimaru H, Matsubara H, Uchida T, Watanabe A, Imakiire T, Nishida Y, and Kumagai H (2017) Direct effects of glucose, insulin, GLP-1, and GIP on bulbospinal neurons in the rostral ventrolateral medulla in neonatal wistar rats. Neuroscience 344:74-88.

Pilowsky PM and Godchild AK (2002) Baroreceptor reflex pathways and neurotransmitters: 10 years on. J Hypertens 20:1675-1688.

Rezq S and Abdel-Rahman AA (2016) Rostral ventrolateral medulla EP3 receptor mediates the sympathoexcitatory and pressor effects of prostaglandin E2 in conscious rats. J Pharmacol Exp Then 359:290-299.

Ruginsk SG, Mecawi AS, da Silva MP, Rein WL, Coletti R, de Lima JB, Elias LL, and Antunes-Rodrigues J (2015) Gaseous modulators in the control of the hypothalamic neurohypophyseal system. Physiology (Bethesda) 30:127-138.

Spitz DR, Dewey WC, and Li GC (1987) Hydrogen peroxide or heat shock induces resistance to hydrogen peroxide in Chinese hamster fibroblasts. J Cell Physiol 131:364-373.

Szczepanska-Sadowska E, Cudnoch-Jedrzejewska A, Ufnal M, and Zera T (2010) Brain and cardiovascular diseases: common neurogenic background of cardiovascular, metabolic and inflammatory diseases. J Physio Pharmacol 61:509-521.

van den Born JC, Homes HP, Greffrath W, van Goon H, and Hillebrands JL; DFG GRK International Research Training Group 1874 Diabetic Microvascular Complications (DIAMICOM) (2016) Gasotransmitters in vascular complications of diabets. Diabetes 65:331-345.
Velasco-Xolalpa ME, Barragán-Iglesias P, Roa-Coria JE, Godínez-Chaparro B, Flores-Murrieta FJ, Torres-López JE, Araiza-Saldaña CI, Navarrete A, and RochaGonzález HI (2013) Role of hydrogen sulfide in the pain processing of non-diabetic and diabetic rats. Neuroscience 250:786-797.

Wang JQ, Yin J, Song YF, Chang L, Run YX, Wang DG, Gao LP, and Sing YH (2014) Brain aging and $\mathrm{AD}$-like pathology in streptozotocin-induced diabetic rats. $J \mathrm{Di}$ abeles Res 2014:796840.

Wang X and Abdel-Rahman AA (2002) Estrogen modulation of eNOS activity and its association with caveolin-3 and calmodulin in rat hearts. Am J Physio Heart Cire Physio 282:H2309-H2315.

Wang X and Abdel-Rahman AA (2005) Effect of chronic ethanol administration on hepatic eNOS activity and its association with caveolin-1 and calmodulin in female rats. Am J Physio Gastrointest Liver Physio 289:G579-G585.

Yon YH, Chou CCK, Wang JS, Pung CL, Li YR, Lo K, and Ching TJ (2014) Subchronic effects of inhaled ambient particulate matter on glucose homeostasis and target organ damage in a type 1 diabetic rat model. Toxicol Appl Pharmacol 281: 211-220.

Yang LY, Thu YH, Tweedie D, Yo QS, Pick CG, Hoffer BJ, Grig NH, and Wang JY (2015) Post-trauma administration of the pifithrin- $\alpha$ oxygen analog improves histological and functional outcomes after experimental traumatic brain injury. Exp Neural 269:56-66.

Zimmerman MC and Davisson RL (2004) Redox signaling in central neural regulatimon of cardiovascular function. Prog Biophys Mol Biol 84:125-149.

$\overline{\text { Address correspondence to: Dr. Abdel A. Abdel-Rahman, Department of }}$ Pharmacology, Body School of Medicine, East Carolina University, Greenville, NC 27834. E-mail: abdelrahmana@ecu.edu 